\title{
A phase I study of OSI-461 in combination with mitoxantrone in patients with advanced solid tumors potentially responsive to mitoxantrone
}

\author{
Lee P. Resta - Roberto Pili - Mario A. Eisenberger - Avery Spitz • \\ Serina King · Jennifer Porter • Amy Franke $\cdot$ Ramesh Boinpally \\ Michael A. Carducci · Christopher J. Sweeney
}

Received: 26 March 2010/Accepted: 8 April 2010/Published online: 6 May 2010

(C) The Author(s) 2010. This article is published with open access at Springerlink.com

\begin{abstract}
Purpose To find the maximum tolerated dose (MTD) of OSI-461 in combination with mitoxantrone in patients with advanced solid tumors.

Methods This was a Phase I study using cohort dose escalation of OSI-461 dosed orally twice daily in combination with mitoxantrone $12 \mathrm{mg} / \mathrm{m}^{2}$ given on Day 1 of each 21-day cycle.

Results OSI-461 dose was escalated to $1,000 \mathrm{mg}$ po bid. One patient experienced a dose-limiting toxicity (DLT). Three patients discontinued the study due to adverse events (AE). Two patients (10\%) had a partial response, and ten patients $(50 \%)$ had stable disease as best response.

Conclusion The combination of OSI-461 and mitoxantrone was well tolerated. Dose escalation was stopped because of toxicities in a concurrent Phase I trial. The response rate seen in patients with prostate cancer was comparable to response rates seen in trials of mitoxantrone and prednisone alone, and further studies of the combination of OSI-461 and mitoxantrone were not pursued.
\end{abstract}

Keywords Mitoxantrone - OSI-461 - Apoptosis · Clinical trial

L. P. Resta - R. Pili - M. A. Eisenberger · A. Spitz - S. King

M. A. Carducci $(\square)$

The Sidney Kimmel Comprehensive Cancer

Center at Johns Hopkins, 1650 Orleans Street,

CRB1-1M59, Baltimore, MD 21231, USA

e-mail: carducci@jhmi.edu

A. Franke $\cdot$ R. Boinpally

OSI Pharmaceuticals Inc., Melville, NY, USA

J. Porter · C. J. Sweeney

Indiana University Simon Cancer Center,

Indianapolis, IN, USA

\section{Introduction}

OSI-461 is a second generation selective apoptotic antineoplastic drug (SAAND). SAANDs induce apoptosis of tumor cells by inhibiting $3^{\prime}, 5^{\prime}$-cyclic guanosine monophosphate (cGMP) phosphodiesterase (PDE) isoforms PDE2 and PDE5, elevating cGMP, activating protein kinase $\mathrm{G}(\mathrm{PKG})$ and decreasing $\beta$-catenin [1]. OSI-461 has approximately $100 \times$ more affinity for cGMP PDE than does exisulind, a first generation SAAND compound.

In vitro data suggest that OSI-461 inhibits angiogenesis and blocks mitotic progression through disruption of microtubule organization and spindle formation [2]. OSI461 induces apoptosis in a wide variety of epithelialderived and non-epithelial-derived tumor cell lines while sparing normal cells. Nude mice with human prostate adenocarcinoma xenografts showed a decrease in tumor size when treated with mitoxantrone and OSI-461 when compared to animals treated with either mitoxantrone or OSI-461 alone.

Mitoxantrone is a commercially available synthetic cytotoxic antineoplastic anthracenedione derivative approved by the FDA and used routinely in combination with corticosteroids for the treatment of patients with advanced hormone-refractory prostate cancer. It has also been extensively studied in the treatment of breast cancer, leukemia and lymphoma. Mitoxantrone causes crosslinks and strand breaks in DNA, interferes with RNA and inhibits topoisomerase II.

OSI-461 is a cytostatic agent that has shown modest antitumor activity in a Phase II pilot study involving patients with hormone-refractory prostate cancer. The combination of cytostatic agents with standard cytotoxic therapies is under intensive evaluation, and the combination of OSI-461 and mitoxantrone, which showed evidence 
of efficacy in a preclinical model as described above, appeared worthwhile to investigate both from a pharmacologic and from a clinical perspective. In addition, both agents have demonstrated activity in patients with hormone-refractory prostate cancer. Therefore, we conducted a Phase I dose-finding study of OSI-461 orally twice daily in combination with mitoxantrone dosed on Day 1 of each 21-day cycle.

\section{Patients and methods}

\section{Study design}

This was a two-center, open-label Phase I study using a $3+3$ cohort dose escalation design to determine the maximum tolerated dose (MTD) of OSI-461 po bid that can be given in combination with mitoxantrone to patients with advanced solid tumors. Secondary objectives included describing the pharmacokinetic (PK) data and toxicity profiles (including any correlations between these profiles) and evaluating the anticancer activity of OSI-461 and mitoxantrone in combination. This study was approved by the appropriate Institutional Review Boards, and all enrolled patients provided written informed consent.

\section{Patient selection}

Patients were eligible for this study if they met all of the following inclusion criteria: histologically documented solid tumor (measurable or non-measurable) potentially responsive to mitoxantrone and for which no effective therapy was available; age $\geq 18$ years; ECOG performance status $0-2$; predicted life expectancy $\geq 12$ weeks. Patients may have had any number of prior chemotherapy or radiation therapy regimens, but a minimum of 4 weeks must have elapsed between the end of previous therapy and entry into the protocol. Patients previously exposed to anthracyclines may not have exceeded a cumulative anthracycline dose of $250 \mathrm{mg} / \mathrm{m}^{2}$ of Adriamycin. Patients were required to have adequate bone marrow, hepatic and renal function as defined by the following: neutrophil count $\geq 1.5 \times 10^{9} / \mathrm{L}$; platelets $100 \times 10^{9} / \mathrm{L}$; total bilirubin $\leq$ upper limit of normal; ALT and AST $\leq 2.5 \times$ upper limit of normal; and serum creatinine $\leq 2.0 \mathrm{mg} / \mathrm{dL}$. Patients were also required to have adequate cardiac function with an estimated left ventricular ejection fraction of $\geq 50 \%$. Patients were excluded if they had a history of previous myocardial infarction, cerebrovascular accident or uncontrolled atrial fibrillation within 1 year of screening. Concurrent use of prednisone or luteinizing hormone-releasing hormone (LHRH) was permissible.
Treatment plan and dose escalation

The starting dose of OSI-461 was $200 \mathrm{mg}$ po taken once on Cycle 1, Day 1 and twice daily from Day 2 onward. Mitoxantrone was given at $12 \mathrm{mg} / \mathrm{m}^{2}$ as a $30-\mathrm{min}$ IV infusion starting on Cycle 1, Day 1 and repeated on Day 1 of every 21-day cycle. On Day 1 of Cycles 1 and 2, patients were instructed to eat a high-fat, high-calorie meal within 30 min of the scheduled dosing time. On other days of the study, patients took OSI-461 with 8 oz of water and within $30 \mathrm{~min}$ of eating.

An initial cohort of three patients was treated at each dose level. Dose escalation did not occur until the last patient treated in the previous cohort had been observed for one complete cycle of therapy. If no patients in a given cohort experienced a dose-limiting toxicity (DLT), the OSI-461 dose was escalated by $200 \mathrm{mg}$ bid. If one patient in a given cohort experienced a DLT, three additional patients were enrolled at the same dose of OSI-461 and observed. If no additional DLTs were observed, then dose escalation continued. Once a second patient in a given cohort experienced a DLT, dose escalation was stopped, and the MTD was to be defined as the dose level below which $\geq 33 \%$ of patients experienced a DLT. The MTD was expanded to a maximum of 10 patients to further evaluate safety and pharmacokinetics at this dose level.

Toxicities were graded according to the National Cancer Institute Common Terminology Criteria for Adverse Events v3.0 (NCI CTCAE). A DLT was any toxicity that was considered at least possibly related to protocol therapy and was experienced during the first cycle of therapy. DLT was defined as the following: $\geq$ grade 3 non-hematologic toxicity (excluding inadequately treated nausea, vomiting and/or diarrhea), grade 4 neutropenia for $\geq 7$ days, febrile neutropenia (grade 3 neutropenia associated with fever $\geq 38.5^{\circ} \mathrm{C}$ ), grade 4 thrombocytopenia or bleeding requiring a platelet transfusion, or treatment delay of 14 days or greater because of treatment-related toxicity. Patients experiencing a DLT were permitted to continue treatment at the next lower dose level of OSI-461.

Doses were also adjusted or delayed for toxicities. If a patient experienced several toxicities, dose adjustments were made according to the system showing the greatest degree of toxicity (reducing the dose to the lowest level). OSI-461 dose was held for $\geq$ grade 3 elevated transaminases or $\geq$ grade 1 bilirubinemia. OSI-461 dose was decreased $50 \%$ for grade 2 elevated transaminases. Mitoxantrone was discontinued for decreases in left ventricular ejection fraction of $10 \%$ or greater. Mitoxantrone dose was reduced one dose level for grade 4 neutropenia, febrile neutropenia, documented infection with Grade 3/4 neutropenia or grade 4 thrombocytopenia. Mitoxantrone was held for up to 14 days for grade 2 elevated 
transaminases or grade 1 bilirubinemia. OSI-461 and mitoxantrone were held for other grade 3 non-hematologic toxicities until these resolved, and OSI-461 and mitoxantrone doses were then decreased by one dose level.

Patients were continued on protocol in the absence of disease progression or unacceptable toxicity. Study medication was discontinued in the event of disease progression, unacceptable toxicity, patient death, patient request or for medical/ethical reasons.

\section{Study procedures}

Prior to enrollment, patients underwent a complete medical history review, physical exam, laboratory assessment, and MUGA or echocardiogram. A repeat physical examination was done on Day 1 of each subsequent cycle. Laboratory assessments were obtained weekly during Cycles 1 and 2 and on Days 1 and 8 of subsequent cycles. MUGA or echocardiogram was repeated every second cycle. Tumor evaluations were done at baseline and then after every second cycle. Response and progression were evaluated using the Response Evaluation Criteria in Solid Tumors (RECIST) guidelines [3]. Prostate-specific antigen (PSA) was measured at baseline and at the beginning of each cycle of treatment for all patients with prostate cancer. Time to PSA progression as defined by the Prostate Cancer Clinical Trials Working Group (PCWG2) was calculated when possible [4]. All patients who received at least one dose of OSI-461 were included in the safety analysis. All patients who received at least one cycle of therapy and had their disease re-evaluated were included in the efficacy analysis.

\section{Pharmacokinetics}

Blood samples for pharmacokinetic (PK) analysis were drawn on Day 1 (predose OSI-461 and preinfusion mitoxantrone, immediately after mitoxantrone infusion, and 15, $30 \mathrm{~min}, 1,2,4,7$ and $9 \mathrm{~h}$ postinfusion), Day 2 (24 h postinfusion and predose OSI-461), Day 3 or 4 (morning), Day 5 or 6 (morning), and Day 8 (morning) of Cycles 1 and 2. An additional blood sample was collected on Day 22 (predose OSI-461 and preinfusion mitoxantrone) of Cycle 2.

Plasma from heparinized blood samples was analyzed by a validated liquid chromatography/mass spectrometry method. Pharmacokinetic parameters of OSI-461 and mitoxantrone were computed for each patient by noncompartmental analyses using WinNonlin ${ }^{\circledR} 5.2$ (Pharsight Corporation, Mountain View, CA). Actual blood collection times were used in constructing the tables of individual plasma concentrations of OSI-461 and mitoxantrone and for calculation of PK parameters.

\section{Results}

Patient characteristics

The demographic characteristics of the patients enrolled in the study are shown in Table 1. Most patients had received two or more chemotherapy regimens as well as radiation therapy. One patient was enrolled but never treated because of decline in performance status prior to the first cycle of treatment.

\section{Dosing}

Three patients each were treated at OSI-461 200, 400, 600 and $800 \mathrm{mg}$ po bid without DLT. Eight patients were treated at OSI-461 1,000 mg po bid. This was the maximum dose level studied in this trial because of toxicities seen in a concurrent Phase I study of single-agent OSI-461. Patients were on study for a mean of 57, 74, 69, 179 and 70 days at OSI-461 200, 400, 600, 800 and 1,000 mg po bid, respectively.

Beyond cycle 1, six patients (30\%) had their OSI-461 doses modified, including two patients at OSI-461 $600 \mathrm{mg}$ po bid, one patient at OSI-461 $800 \mathrm{mg}$ po bid and three patients at OSI-461 1,000 mg po bid. Reasons for OSI-461 dose modifications included elevated bilirubin (two patients), neutropenia (two patients) and elevated liver enzymes and rash (one patient each).

The total number of mitoxantrone cycles was 10, 10, 9, 19 and 26 at OSI-461 200, 400, 600, 800 and 1,000 mg po

Table 1 Patient characteristics

\begin{tabular}{ll}
\hline Entered/treated & $21 / 20$ \\
Male/female & $16 / 4$ \\
Median age in years (range) & $61(48-81)$ \\
ECOG PS & \\
0 & $7(35 \%)$ \\
1 & $10(50 \%)$ \\
2 & $2(10 \%)$ \\
Missing & $1(5 \%)$ \\
Primary tumor & \\
Prostate & $14(70 \%)$ \\
Breast & $4(20 \%)$ \\
Bladder & $1(5 \%)$ \\
Testicular & $1(5 \%)$ \\
Prior chemotherapy regimens & \\
$0-1$ & 6 \\
$2-3$ & 9 \\
$4+$ & 5 \\
Prior radiotherapy, yes/no & $15 / 5$ \\
\hline
\end{tabular}


bid, respectively. There were no mitoxantrone dose reductions at OSI-461 200, 600 or $800 \mathrm{mg}$ po bid. There was one mitoxantrone dose reduction at OSI-461 $400 \mathrm{mg}$ po bid in cycle 6 for grade 4 neutropenia. There were two mitoxantrone dose reductions at OSI-461 $1,000 \mathrm{mg}$ po bid, one in cycle 2 due to grade 3 rash and one in cycle 3 due to grade 4 neutropenia.

\section{Toxicities and adverse events}

Twenty patients (100\%) experienced at least one adverse event, and 17 patients (85\%) experienced an adverse event considered related to OSI-461 and/or mitoxantrone. The most common AEs included GI events and fatigue, which were generally of severity grade 1 or 2 . A summary of AEs and toxicities reported for all cycles is shown in Table 2 . There were two treatment-related grade three adverse events: hearing loss in one patient in the OSI-461 $800 \mathrm{mg}$ po bid cohort and one in cycle 2 due to grade 3 erythematous rash in one patient at OSI-461 $1,000 \mathrm{mg}$ po bid. Another patient at OSI-461 1,000 mg po bid experienced a DLT (grade 4 neutropenia for $\geq 7$ days) that was considered to be due to mitoxantrone. Eight patients experienced at least one serious adverse event (SAE), none of which were related to study drug.

Most patients experienced hematologic laboratory abnormalities that did not qualify as a DLT, including $70 \%$ of patients with grade $3 / 4$ lymphopenia and $65 \%$ of patients with grade $3 / 4$ neutropenia and leukopenia. No patients had febrile neutropenia. The median duration of grade 3 or grade 4 leukopenia was 7 days (range 342 days). Fifty five percent of patients experienced transaminase elevation, though this was mostly low grade and transient. One patient at OSI-461 $1,000 \mathrm{mg}$ po bid had the OSI-461 dose reduced by $50 \%$ due to grade 2 ALT/AST, but the ALT continued to increase to grade 3 at which time the patient was taken off study due to progressive disease. Three patients (15\%) experienced an absolute LVEF decrease of $10 \%$ or greater: one patient at OSI-461 $400 \mathrm{mg}$ po bid had a $12 \%$ decrease after 2 cycles; one patient at OSI-461 $1,000 \mathrm{mg}$ po bid had a $12 \%$ decrease after six cycles; and one patient at OSI-461 $1,000 \mathrm{mg}$ po bid had a $29 \%$ decrease after eight cycles (this patient's LVEF returned to normal after discontinuing mitoxantrone).

Three AEs resulted in patient discontinuation from the study. One patient at OSI-4611,000 $\mathrm{mg}$ po bid had a treatment-related SAE (Grade 3 rash) in Cycle 2 that resulted in discontinuation. Two additional patients had non-treatment-related AEs that resulted in discontinuation, including one patient at OSI-461 $200 \mathrm{mg}$ po bid with hyponatremia and another patient at OSI-461 $400 \mathrm{mg}$ po bid with pneumonia.
Disease response

Twelve of the 20 treated patients $(60 \%)$ had either stable disease (10 patients) or a partial response (two patients) as their best measured response by RECIST guidelines. One patient with prostate cancer whose disease had previously progressed on hormonal therapy, docetaxel and estramustine had a confirmed partial response after cycle 8 at OSI$461400 \mathrm{mg}$ po bid. One patient with breast cancer whose disease had previously progressed on hormonal therapy and five different chemotherapy regimens (including two investigational agents) had a partial response after cycle 8 at OSI-461 $800 \mathrm{mg}$ po bid. The range of duration of stable disease was two to eight cycles.

Fourteen patients had evidence of progressive disease at the time of discontinuation from the study. The overall median time to progression from date of first treatment was 48 days. The median times to progression for patients treated at OSI-461 $200 \mathrm{mg}$ po bid, $400 \mathrm{mg}$ po bid, $600 \mathrm{mg}$ po bid, $800 \mathrm{mg}$ po bid and $1,000 \mathrm{mg}$ po bid were 33,53 , 53, 236 days (one patient) and 52 days, respectively.

Seven of the 14 patient with prostate cancer patients (50\%) experienced either a partial response (one patient) or stable disease (six patients) per RECIST as their best response at their first planned imaging after two cycles. The median time to disease progression for patients with prostate cancer was 48 days. All four of the patients with breast cancer had a partial response (one patient) or stable disease (three patients) as their best response.

Two of the 14 patients with prostate cancer $(14 \%)$ treated had a PSA response (decreases in their PSA levels of $50 \%$ or more when compared to pretreatment baseline levels). One of these patients was treated at OSI-461 $400 \mathrm{mg}$ po bid and had previous disease progression on hormonal therapy and chemotherapy (docetaxel and estramustine), and the other was treated at OSI-461 1,000 mg po bid and also had previous disease progression on hormonal therapy and chemotherapy (amonafide and docetaxel).

Ten patients with prostate cancer did not have decreases in their PSA levels and did not remain on trial at least 12 weeks as required to calculate time to PSA progression as defined by the PCWG2. The four patients with prostate cancer who experienced any decline in their PSA levels had times to PSA progression of 63 days (not confirmed), 84 days (confirmed), 120 days (confirmed) and 169 days (confirmed).

\section{Pharmacokinetics}

OSI-461 peak plasma concentration and exposure increased linearly in proportion to dose within the OSI-461 dose range tested (200-1,000 mg po bid). As detailed in Fig. 1a and Table 3, there were no significant differences 
Table 2 Adverse events and laboratory toxicities by maximum grade for all cycles

\begin{tabular}{|c|c|c|c|c|c|c|c|c|c|c|c|c|}
\hline & \multicolumn{10}{|c|}{ OSI-461 Dose (PO bid) } & \multirow{2}{*}{\multicolumn{2}{|c|}{ Total }} \\
\hline & \multicolumn{2}{|c|}{$200 \mathrm{mg}$} & \multicolumn{2}{|c|}{$400 \mathrm{mg}$} & \multicolumn{2}{|c|}{$600 \mathrm{mg}$} & \multicolumn{2}{|c|}{$800 \mathrm{mg}$} & \multicolumn{2}{|c|}{$1000 \mathrm{mg}$} & & \\
\hline & All & $3 / 4$ & All & $3 / 4$ & All & $3 / 4$ & All & $3 / 4$ & All & $3 / 4$ & All & $3 / 4$ \\
\hline \multicolumn{13}{|c|}{ All AEs (regardless of causality) } \\
\hline Fatigue & 2 & 0 & 1 & 0 & 2 & 0 & 1 & 0 & 4 & 1 & $10(50 \%)$ & $1(5 \%)$ \\
\hline Nausea & 2 & 0 & 2 & 0 & 3 & 0 & 2 & 0 & 1 & 0 & $10(50 \%)$ & 0 \\
\hline Back pain & 1 & 0 & 2 & 0 & 1 & 1 & 2 & 0 & 1 & 1 & $7(35 \%)$ & $2(10 \%)$ \\
\hline Diarrhea & 1 & 0 & 1 & 0 & 1 & 0 & 1 & 0 & 3 & 0 & $7(35 \%)$ & 0 \\
\hline Arthralgia & 1 & 1 & 1 & 0 & 1 & 1 & 1 & 0 & 2 & 0 & $6(30 \%)$ & $2(10 \%)$ \\
\hline Constipation & 1 & 0 & 1 & 0 & 1 & 0 & 0 & 0 & 3 & 0 & $6(30 \%)$ & 0 \\
\hline Vomiting & 2 & 0 & 1 & 0 & 2 & 0 & 0 & 0 & 0 & 0 & $5(25 \%)$ & 0 \\
\hline Cough & 0 & 0 & 2 & 0 & 1 & 0 & 1 & 0 & 1 & 0 & $5(25 \%)$ & 0 \\
\hline Decreased appetite & 1 & 1 & 0 & 0 & 1 & 0 & 1 & 0 & 1 & 0 & $4(20 \%)$ & $1(5 \%)$ \\
\hline Dysgeusia & 0 & 0 & 0 & 0 & 0 & 0 & 3 & 0 & 1 & 0 & $4(20 \%)$ & 0 \\
\hline Anorexia & 0 & 0 & 0 & 0 & 0 & 0 & 1 & 0 & 3 & 0 & $4(20 \%)$ & 0 \\
\hline Dehydration & 1 & 0 & 1 & 0 & 1 & 0 & 0 & 0 & 1 & 0 & $4(20 \%)$ & 0 \\
\hline Headache & 0 & 0 & 1 & 0 & 0 & 0 & 3 & 0 & 0 & 0 & $4(20 \%)$ & 0 \\
\hline \multicolumn{13}{|l|}{ Treatment-related AEs } \\
\hline Nausea & 2 & 0 & 2 & 0 & 3 & 0 & 1 & 0 & 0 & 0 & $8(40 \%)$ & 0 \\
\hline Fatigue & 1 & 0 & 1 & 0 & 1 & 0 & 1 & 0 & 2 & 0 & $6(30 \%)$ & 0 \\
\hline Diarrhea & 0 & 0 & 1 & 0 & 1 & 0 & 1 & 0 & 2 & 0 & $5(25 \%)$ & 0 \\
\hline Vomiting & 2 & 0 & 1 & 0 & 2 & 0 & 0 & 0 & 0 & 0 & $5(25 \%)$ & 0 \\
\hline Constipation & 1 & 0 & 0 & 0 & 1 & 0 & 0 & 0 & 2 & 0 & $4(20 \%)$ & 0 \\
\hline Dysgeusia & 0 & 0 & 0 & 0 & 0 & 0 & 3 & 0 & 1 & 0 & $4(20 \%)$ & 0 \\
\hline \multicolumn{13}{|l|}{ Laboratory toxicities } \\
\hline Neutropenia & 2 & 1 & 3 & 1 & 3 & 2 & 3 & 3 & 8 & 6 & $19(95 \%)$ & $13(65 \%)$ \\
\hline Leukopenia & 3 & 1 & 3 & 1 & 3 & 3 & 3 & 3 & 8 & 5 & $20(100 \%)$ & $13(65 \%)$ \\
\hline Lymphopenia & 3 & 1 & 2 & 2 & 3 & 3 & 3 & 2 & 8 & 6 & $19(95 \%)$ & $14(70 \%)$ \\
\hline Transaminitis & 1 & 0 & 2 & 0 & 2 & 0 & 1 & 0 & 5 & 1 & $11(55 \%)$ & $1(5 \%)$ \\
\hline Bilirubinemia & 1 & 0 & 0 & 0 & 1 & 0 & 0 & 0 & 3 & 0 & $5(25 \%)$ & 0 \\
\hline
\end{tabular}

between the different AUC parameters of OSI 461 from Cycle 1 to Cycle 2, indicating a lack of accumulation at steady state. Also, there was no significant relationship between dosing level and clearance rate or half-life. Finally, the pharmacokinetic parameters for mitoxantrone were not significantly affected by OSI-461 at any dose level tested (Fig. 1b and Table 4).

\section{Discussion}

OSI-461 is a second generation SAAND, a class of antineoplastic drugs that induce apoptosis by inhibiting cGMP phosphodiesterase isoforms PDE2 and PDE5. Exisulind was a first generation SAAND compound and was extensively studied in combination with chemotherapy in a variety of tumor types, including prostate cancer. A Phase I/II study of oral exisulind twice daily in combination with docetaxel given in a 3-week cycle was performed in patients with hormone-refractory prostate cancer [5]. The PSA response rate for patients treated with the Phase II dose was only $23 \%$, and the combination was not explored further. Another Phase II trial explored the combination of twice daily oral exisulind in combination with weekly docetaxel in patients with hormone-refractory prostate cancer [6]. Approximately $20 \%$ of patients had a PSA response, and this combination regimen was also not explored further. Additional Phase II studies of exisulind in combination with chemotherapy in lung cancer also failed to show sufficient efficacy to warrant further study [7-9].

OSI-461 has approximately $100 \times$ more affinity for cGMP PDE than does exisulind, a first generation SAAND compound. OSI-461 inhibited the growth of prostate cancer cell lines in vitro, and in a pilot Phase II study, OSI-461 showed modest antitumor activity in patients with hormone-refractory prostate cancer [10]. Mitoxantrone is 
Fig. 1 Median plasma OSI-461 concentrations at each OSI-461 dose level for Cycles 1 and 2 (a) and the median plasma mitoxantrone concentrations at each OSI-461 dose level for Cycles 1 and 2 (b)
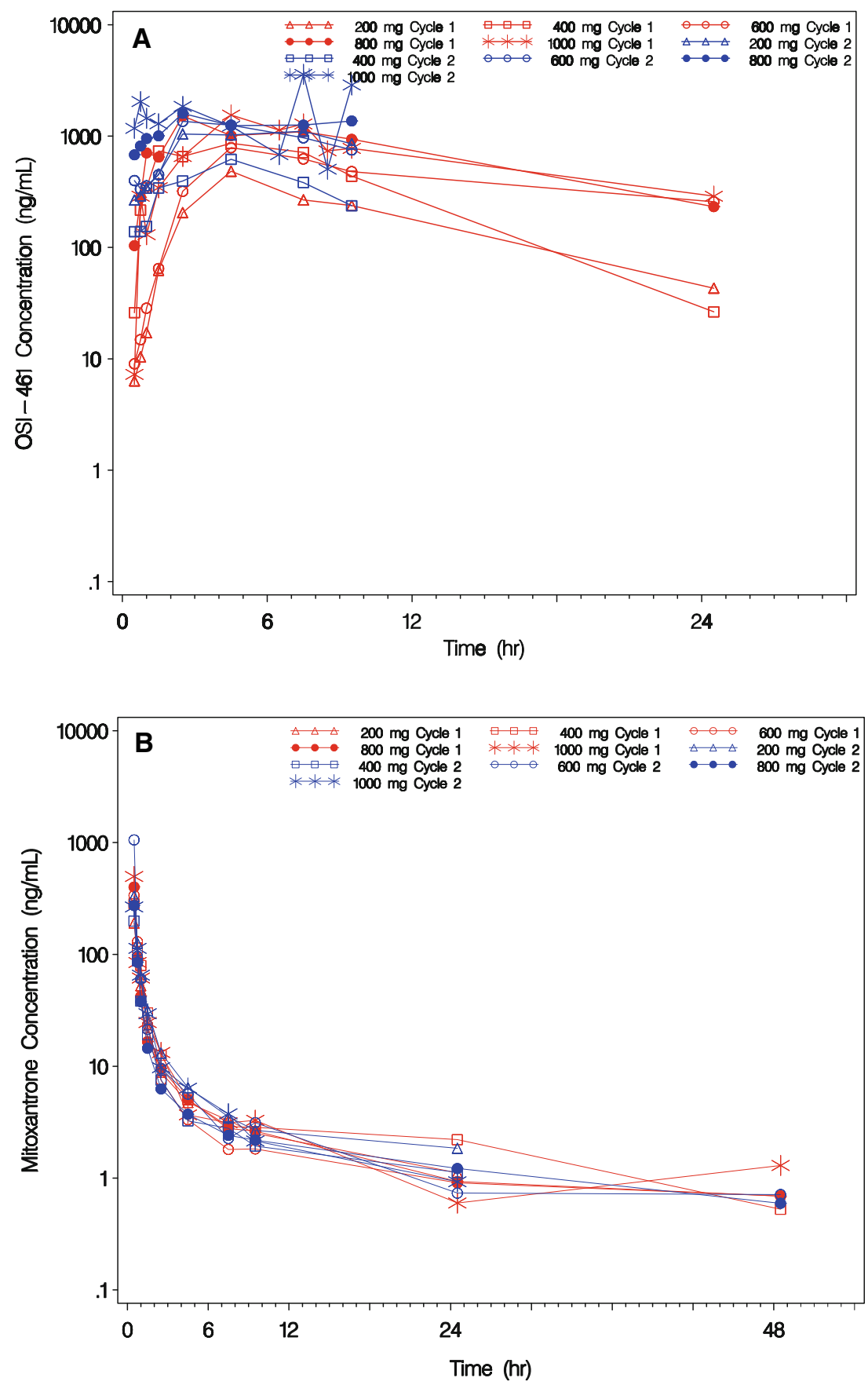

commonly used in combination with corticosteroids for the treatment of hormone-refractory prostate cancer and is also used in other tumor types, including breast cancer. Thus, we conducted a Phase I dose-finding study of OSI-461 given orally twice daily in combination with mitoxantrone dosed on Day 1 of a 21-day cycle.

The combination regimen explored in this study was well tolerated, and only one DLT was observed at the highest OSI-461 dose level studied (Dose Level 4, OSI-461 $1,000 \mathrm{mg}$ po bid). Of the three AEs that resulted in patient discontinuation from the study, only one (Grade 3 rash) was felt to be treatment related. The most common AEs included GI events and fatigue, and all of these were $\leq$ Grade 2 in severity. Severe hematologic toxicities such as grade 3/4 neutropenia, leukopenia and lymphopenia were common, but no episodes of febrile neutropenia were observed.

Dose escalation was not continued above OSI-461 $1,000 \mathrm{mg}$ po bid because of toxicities seen in a concurrent Phase I study of single-agent OSI-461 in patients with advanced solid tumors. In that study, three DLTs of Grade 3 abdominal pain were seen at the OSI-461 dose level of 
Table 3 Summary of pharmacokinetic parameters of OSI-461 (Cycle 1)

\begin{tabular}{|c|c|c|c|c|c|c|c|}
\hline \multirow[t]{2}{*}{ Parameter } & \multicolumn{7}{|c|}{ OSI-461 Dose (PO bid) } \\
\hline & & $200 \mathrm{mg}$ & $400 \mathrm{mg}$ & $600 \mathrm{mg}$ & $800 \mathrm{mg}$ & $1,000 \mathrm{mg}$ & All \\
\hline \multirow[t]{2}{*}{$C_{\max }(\mathrm{ng} / \mathrm{ml})$} & Median & 0.57 & 1.04 & 0.85 & 1.80 & 1.98 & \\
\hline & Range & $0.14-1.00$ & $0.71-1.84$ & $0.42-1.72$ & $1.56-2.26$ & $0.87-4.07$ & $0.14-4.07$ \\
\hline \multirow[t]{2}{*}{$\mathrm{AUC}_{0 \rightarrow \text { inf }}(\mathrm{hr} \cdot \mathrm{ug} / \mathrm{ml})$} & Median & 5.28 & 8.08 & 13.6 & 17.7 & 25.0 & \\
\hline & Range & $2.26-8.29$ & $6.39-13.9$ & $13.6-13.6$ & $12.4-23.0$ & $12.0-73.4$ & $2.26-73.4$ \\
\hline \multirow[t]{2}{*}{$\mathrm{Cl} / \mathrm{F}(\mathrm{L} / \mathrm{hr})$} & Median & 56.3 & 49.5 & 44.0 & 49.7 & 40.0 & \\
\hline & Range & $24.1-88.5$ & $28.8-62.6$ & $44.0-44.0$ & $34.8-64.7$ & $13.6-83.6$ & $13.6-88.5$ \\
\hline \multirow[t]{2}{*}{$t_{1 / 2}(\mathrm{hr})$} & Median & 6.18 & 4.94 & 16.9 & 7.54 & 4.81 & \\
\hline & Range & $4.93-44.7$ & $3.45-5.12$ & $4.88-26.3$ & $4.33-129$ & $3.58-129$ & $3.45-129$ \\
\hline
\end{tabular}

Table 4 Summary of pharmacokinetic parameters of mitoxantrone (Cycle 1)

\begin{tabular}{|c|c|c|c|c|c|c|c|}
\hline \multirow[t]{2}{*}{ Parameter } & \multicolumn{7}{|c|}{ OSI-461 Dose (PO bid) } \\
\hline & & $200 \mathrm{mg}$ & $400 \mathrm{mg}$ & $600 \mathrm{mg}$ & $800 \mathrm{mg}$ & $1,000 \mathrm{mg}$ & All \\
\hline \multirow[t]{2}{*}{$C_{\max }(\mathrm{ng} / \mathrm{ml})$} & Median & 191 & 229 & 323 & 593 & 386 & 318 \\
\hline & Range & 94-306 & $128-427$ & $256-391$ & $380-1,372$ & $214-538$ & $94-1,372$ \\
\hline \multirow[t]{2}{*}{$\mathrm{AUC}_{0 \rightarrow \text { inf }}(\mathrm{hr} \cdot \mathrm{ng} / \mathrm{ml})$} & Median & 315 & 360 & 253 & 294 & 303 & 298 \\
\hline & Range & $287-343$ & $119-403$ & $200-322$ & $256-714$ & $210-525$ & $119-714$ \\
\hline \multirow[t]{2}{*}{$\mathrm{Cl}\left(\mathrm{L} / \mathrm{hr} / \mathrm{m}^{2}\right)$} & Median & 38.4 & 33.3 & 47.4 & 40.8 & 39.7 & 40.2 \\
\hline & Range & $35-42$ & $30-101$ & $37-60$ & $17-47$ & $23-58$ & $17-101$ \\
\hline \multirow[t]{2}{*}{$t_{1 / 2}(\mathrm{hr})$} & Median & 34.8 & 16.1 & 20.1 & 17.0 & 10.0 & 17.0 \\
\hline & Range & $10-47$ & $6-24$ & $3-21$ & $17-18$ & $6-30$ & $3-47$ \\
\hline
\end{tabular}

$1200 \mathrm{mg}$ po bid. This was thought to be due to gelatin accumulation in the gastrointestinal tract from the large number of capsules ingested (24 capsules per day). Two additional DLTs (Grade 3 abdominal pain and Grade 3 fatigue) were seen in the single-agent Phase I study at the OSI-461 dose level of 1,000 mg po bid, and the MTD of OSI-461 for that study was determined to be $800 \mathrm{mg}$ po bid.

While the primary objective of this study was to find the MTD of the combination of OSI-461 dosed twice daily with mitoxantrone dosed on Day 1 of each 21-day cycle, a secondary objective was to evaluate the activity of this regimen. While 12 of the 20 patients $(60 \%)$ treated had either stable disease or a partial response as their best measured response, only five $(25 \%)$ had stable disease or a partial response that persisted beyond the first two cycles of treatment.

Fourteen of the patients enrolled in this study had prostate cancer, and only four (29\%) of these patients had decreases in their PSA levels. Of these four patients, only two had a PSA response (decrease of $\geq 50 \%$ from their baseline levels). Of the eight patients treated at the highest dose level (Dose Level 4, OSI-461 1,000 mg po bid), seven had prostate cancer. Only two of these patients (29\%) had a PSA response. For comparison, mitoxantrone and prednisone demonstrated a PSA response in $32 \%$ and $27 \%$ of patients in the TAX 327 and SWOG 9916 trials, respectively [11, 12]. Thus, the responses seen in this study may have been due to mitoxantrone and not the combination of OSI-461 and mitoxantrone. Based on the results of this study, in addition to the results of the TAX 327 and SWOG 9916 trials, further investigation into the combination of twice daily OSI-461 in combination with mitoxantrone given every 3 weeks was not pursued.

Acknowledgment This work was financially supported by OSI Pharmaceuticals Inc.

Open Access This article is distributed under the terms of the Creative Commons Attribution Noncommercial License which permits any noncommercial use, distribution, and reproduction in any medium, provided the original author(s) and source are credited.

\section{References}

1. Thompson WJ, Piazza GA, Li H et al (2000) Exisulind induction of apoptosis involves guanosine $3^{\prime}, 5^{\prime}$-cyclic monophosphate phosphodiesterase inhibition, protein kinase $\mathrm{G}$ activation, and attenuated beta-catenin. Cancer Res 60:3338-3342

2. Whitehead CM, Earle KA, Xu S et al (2002) CP461 in an orthotopic human NSCLC rat model involves phosphodiesterase 
targeting, apoptosis induction, G2/M block and anti-proliferation. Proc Am Assoc Cancer Res 43:924

3. Therasse P, Arbuck SG, Eisenhauer EA et al (2000) New guidelines to evaluate the response to treatment in solid tumors. $\mathrm{J}$ Natl Cancer Inst 92(3):205-216

4. Scher HI, Halabi S, Tannock I et al (2008) Design and end points of clinical trials for patients with progressive prostate cancer and castrate levels of testosterone: recommendations of the prostate cancer clinical trials working group. J Clin Oncol 26(7):11481159

5. Ryan CW, Stadler WM, Vogelzang NJ (2005) A phase I/II doseescalation study of exisulind and docetaxel in patients with hormone-refractory prostate cancer. BJU Int 95(7):963-968

6. Sinibaldi VJ, Elza-Brown K, Schmidt J et al (2006) Phase II evaluation of docetaxel plus exisulind in patients with androgen independent prostate carcinoma. Am J Clin Oncol 29:395-398

7. Jones SF, Kuhn JG, Greco FA et al (2005) A phase I/II study of exuslind in combination with docetaxel/carboplatin in patients with metastatic non-small cell lung cancer. Clin Lung Cancer 6(6):361-366
8. Masters GA, Li S, Dowlati A et al (2006) A phase II trial of carboplatin and gemcitabine with exuslind (IND \#65, 056) in patients with advanced non-small cell lung cancer: an Eastern Cooperative Oncology Group Study (E1501). J Thorac Oncol $1: 673-678$

9. Hoang T, KyungMann K, Merchant J et al (2006) Phase I/II study of gemcitabine and exisulind as second-line therapy in patients with advanced non-small cell lung cancer. J Thorac Oncol 1:218225

10. Petrylak DP (2003) A phase II study of CP-461 in prostate cancer patients with measurable disease. Cancer Invest 21(Suppl 1):ixxxx 1-108

11. Tannock IF, de Wit R, Berry WR et al (2004) Docetaxel plus prednisone or mitoxantrone plus prednisone for advanced prostate cancer. N Engl J Med 351:1502-1512

12. Petrylak DP, Tangen CM, Hussain MHA et al (2004) Docetaxel plus estramustine compared with mitoxantrone plus prednisone for advanced prostate cancer. N Engl J Med 351:1513-1520 